\title{
Mild Poikilocapnic Hypoxia Increases Very Low Frequency Haemoglobin Oxygenation Oscillations in Prefrontal Cortex
}

\section{Agnieszka Gruszecka}

Medical University of Gdansk: Gdanski Uniwersytet Medyczny

\section{Monika Waskow}

Akademia Pomorska w Slupsku

\section{Marta A. Malkiewicz}

Medical University of Gdansk: Gdanski Uniwersytet Medyczny

\section{J. Patrick Neary}

University of Regina

Jyotpal Singh

University of Regina

\section{Taylor Teckchandani}

University of Regina

\section{Gregory P. Kratzig}

University of Regina

\section{Magdalena Wszedybyl-Winklewska}

Medical University of Gdansk: Gdanski Uniwersytet Medyczny

Andrzej F. Frydrychowski

NIRTI SA, Wroclaw

Jacek Rumiński

Gdańsk University of Technology: Politechnika Gdanska

\section{Natalia Głowacka}

Gdansk University of Technology: Politechnika Gdanska

\section{Piotr Lass}

Medical University of Gdansk: Gdanski Uniwersytet Medyczny

\section{Pawel J. Winklewski}

Medical University of Gdansk: Gdanski Uniwersytet Medyczny

\section{Marcin Gruszecki ( $\nabla$ mgruszecki@gumed.edu.pl )}

Medical University of Gdansk Center for Maritime and Tropical Medicine: Gdanski Uniwersytet Medyczny Centrum Medycyny Morskiej i Tropikalnej https://orcid.org/0000-0003-1709-3939 
Keywords: hypoxia, wavelet transform, blood pressure, subarachnoid space width, near infrared spectroscopy

Posted Date: June 29th, 2021

DOI: https://doi.org/10.21203/rs.3.rs-645557/v1

License: (c) (1) This work is licensed under a Creative Commons Attribution 4.0 International License. Read Full License

Version of Record: A version of this preprint was published at Biological Research on December 1st, 2021. See the published version at https://doi.org/10.1186/s40659-021-00362-2. 


\title{
Mild poikilocapnic hypoxia increases very low frequency haemoglobin oxygenation oscillations in prefrontal cortex
}

\author{
Agnieszka Gruszecka ${ }^{1}$, Monika Waskow², Marta A. Malkiewicz ${ }^{3,4}$, J. Patrick Neary ${ }^{5}$, \\ Jyotpal Singh ${ }^{5}$, Taylor Teckchandani ${ }^{5}$, Gregory P. Kratzig ${ }^{6}$, Magdalena Wszedybyl- \\ Winklewska ${ }^{7}$, Andrzej F. Frydrychowski ${ }^{8}$, Jacek Rumiński ${ }^{9}$, Natalia Głowacka ${ }^{9}$, Piotr \\ Lass $^{10}$, Pawel J. Winklewski ${ }^{7}$, Marcin Gruszecki ${ }^{\star 1,9}$
}

${ }^{1}$ Department of Radiology Informatics and Statistics, Faculty of Health Sciences, Medical University of Gdansk, Gdansk, Poland

${ }^{2}$ Institute of Health Sciences, Pomeranian University of Slupsk, Slupsk, Poland

${ }^{3}$ Applied Cognitive Neuroscience Lab, Department of Human Physiology, Medical University of Gdansk, Gdansk, Poland

${ }^{4}$ Department of Psychiatry, Medical University of Gdansk, Gdansk, Poland

${ }^{5}$ Faculty of Kinesiology and Health Studies, University of Regina, Regina, Canada

${ }^{6}$ Department of Psychology, University of Regina, Regina, SK, Canada

${ }^{7}$ Department of Human Physiology, Faculty of Health Sciences, Medical University of Gdansk, Gdansk, Poland

${ }^{8}$ NIRTI SA, Wroclaw, Poland

${ }^{9}$ Department of Biomedical Engineering, Faculty of Electronics, Telecommunications and Informatics, Gdansk University of Technology, Gdansk, Poland

${ }^{10}$ Department of Nuclear Medicine, Medical University of Gdansk, Gdansk, Poland

${ }^{*}$ Corresponding author:

Marcin Gruszecki, PhD, DSc

Department of Radiology Informatics and Statistics, Medical University of Gdansk

Tuwima Str. 15, 80-210 Gdansk, Poland

E-mail: mgruszecki@gumed.edu.pl; Tel./Fax: +48 58 349-15-08 


\section{Abstract}

The aim of the study was to investigate the effect of mild cerebral hypoxia on haemoglobin oxygenation $\left(\mathrm{HbO}_{2}\right)$, cerebrospinal fluid dynamics and cardiovascular physiology. To achieve this goal, four signals were recorded simultaneously: blood pressure, $\mathrm{ECG}, \mathrm{HbO}_{2}$ from right hemisphere and changes of SAS width from left hemisphere. Signals were registered from 30 healthy, young participants ( 2 females and 28 males, $\mathrm{BMI}=24.5 \pm 2.3 \mathrm{~kg} / \mathrm{m}^{2}$, age $30.8 \pm 13.4$ years). We analysed the recorded signals using wavelet transform (WT). We demonstrated for the first time that in healthy subjects exposed to mild poikilokapnic hypoxia that there were increases in very low frequency $\mathrm{HbO}_{2}$ oscillations $(<0.052 \mathrm{~Hz})$ in prefrontal cortex. Additionally, SAS fluctuation diminishes in the whole frequency range which could be explained by brain oedema. Consequently the study provides insight into mechanisms governing brain response to a mild hypoxic challenge. Our study supports the notion that $\mathrm{HbO}_{2}$ and SAS width monitoring might be beneficial for patients with acute lung disease, including SARS-CoV-2.

\section{Key Words:}

hypoxia, wavelet transform, blood pressure, subarachnoid space width, near infrared spectroscopy

\section{Introduction}

Hypoxia is frequently observed in a number of clinical situations such as lung disease, sleep apnoea, as well as cardiac and cerebrovascular dysfunction associated with aging. Mild hypoxia is also an eminent feature of the respiratory distress syndrome evoked by the coronavirus (SARS-CoV-2). A number of cognitive and neuropsychiatric manifestations of SARS-CoV-2 have been observed in a proportion of patients such as depressed mood, anxiety, post-traumatic stress disorder and cognitive decline. Although these are multifactorial, changes in cerebral oxygen supply and related pathophysiological consequences may play a role (Alonso-Lana et al. 2020).

It is well-known that hypoxia leads to a decline in haemoglobin oxygenation $\left(\mathrm{HbO}_{2}\right)$ in cerebral cortex (Hampson et al. 1990). Diminished oxygen supply to the brain results in several compensatory mechanisms, which in turn trigger substantial changes in 
various aspects of brain functioning. Augmented cerebral blood flow (Kety and Schmidt 1948) and cerebral blood volume (Dubowitz et al. 2009; DiPasquale et al. 2016) together with blood-brain barrier impairment (Halder and Milner 2020) lead to discrete brain oedema and increases in intracranial pressure (Dubowitz et al. 2009; DiPasquale et al. 2016). Furthermore, brain hypoxia results in a number of metabolic changes associated with an augmented cerebral metabolic rate of oxygen, lactate and glutamate concentrations accompanied by a diminished creatinine concentration. Taken together, these metabolic alterations suggest higher neural activity and increased oxidative metabolism (Vestergaard et al. 2016).

The autoregulation dogma is constantly challenged with increasing emphasis on either local neural or metabolic mechanisms (Saleem et al. 2016; Marina et al. 2020). The role of intracranial pressure in the feedback loops regulating widely understood cerebral metabolic homeostasis is continuously highlighted (Winklewski et al. 2019; Marina et al. 2020). Consequently, in this study we aimed at assessing the effect of hypoxia on the oscillatory behaviour of blood pressure (BP), oxygenated haemoglobin $\left(\mathrm{HbO}_{2}\right)$, electrocardiogram (ECG), and subarachnoid space (SAS) width using established non-invasive methods (Ferrari and Quaresima 2012, Gruszecki et al. 2018).

The relative concentrations of the oxygenated and deoxygenated haemoglobin can be measured in the prefrontal area with near infrared spectroscopy (NIRS) (Ferrari and Quaresima 2012). Oscillatory changes in the SAS width, reflecting cerebrospinal fluid pulsatility (Gruszecki et al. 2018), can be instantly monitored with the method developed by our team, called near-infrared transillumination-backscattering sounding (NIR-T/BSS) (Frydrychowski et al. 2002; Frydrychowski et al 2011, Gruszecki et al. 2018). Both methods can be used simultaneously (Gruszecka et al. 2020). Wavelet transform analysis of biological signals can elegantly quantify and delineate the investigated interactions in both frequency and time domains (Stefanovska et al. 1999; Saleem et al. 2016).

We hypothesized that mild hypoxia would increase the prominence of very lowfrequency $\mathrm{HbO}_{2}$ local brain oscillations while maintaining or even decreasing the 
impact of central oscillatory components generated by the heart and lungs. We also expected SAS oscillations to diminish in the whole frequency range as a result of simulated altitude at approximately $2900-3000 \mathrm{~m}$.

\section{Results}

Four signals were continuously and simultaneously recorded from the volunteers: BP, ECG, $\mathrm{HbO}_{2}$ RIGHT and SASLEFT while breathing normal room air $\left(19.8 \% \mathrm{O}_{2}\right)$, and during normobaric hypoxia $\left(14.8 \% \mathrm{O}_{2}\right)$ in an environmental chamber to simulate altitude at 2900-3000m. The atmospheric pressure for whole time of experiment was kept at the same level $712 \mathrm{mmHg}$, while the level of oxygen during normobaric hypoxia was reduced to $14.8 \%$. A gas mixture of $19.8 \% \mathrm{O}_{2}$ was used as the normoxic condition as this is the native partial pressure of oxygen in Regina, Saskatchewan, Canada (577 meters above sea level) where the testing was performed. According to nonparametric Wilcoxon rank sum test (see Table 1) there was a statistically significant drop in amplitude of all measured signals during mild normobaric hypoxia.

\begin{tabular}{|c|c|c|}
\hline & $\begin{array}{c}\text { Gas mixture with } \\
19.8 \% \mathrm{O}_{2}\end{array}$ & $\begin{array}{c}\text { Gas mixture with } \\
14.8 \% \mathrm{O}_{2}\end{array}$ \\
\hline HR [beats/min] & $61.2 \pm 3.6$ & $66.6 \pm 3.4^{\star \star \star}$ \\
\hline DBP [mmHg] & $65.17 \pm 9.65$ & $68.16 \pm 9.34^{*}$ \\
\hline SBP [mmHg] & $112.79 \pm 14.51$ & $119.67 \pm 13.08^{* * *}$ \\
\hline MAP [mmHg] & $81.04 \pm 10.88$ & $85.32 \pm 10.06^{*}$ \\
\hline $\mathrm{SAS}_{\text {LEFT }}[\mathrm{AU}]$ & $0.22 \pm 0.01$ & $0.19 \pm 0.01^{*}$ \\
\hline $\mathrm{HbO}_{2 \text { RIGHT }}\left[\% \mathrm{O}_{2}\right]$ & $0.72 \pm 0.28$ & $0.45 \pm 0.13^{\star \star \star}$ \\
\hline $\mathrm{tHb}[\mu \mathrm{M}]$ & $12.03 \pm 0.43$ & $15.27 \pm 0.51^{* *}$ \\
\hline $\mathrm{SaO}_{2}\left[\% \mathrm{O}_{2}\right]$ & $97.81 \pm 0.61$ & $92.67 \pm 0.97^{\star \star *}$ \\
\hline $\mathrm{EtCO}_{2}[\mathrm{mmHg}]$ & $32.95 \pm 3.16$ & $30.61 \pm 3.25^{\star \star \star}$ \\
\hline $\mathrm{EtO}_{2}[\mathrm{mmHg}]$ & $128.74 \pm 3.29$ & $93.66 \pm 3.67^{* * *}$ \\
\hline
\end{tabular}

Table 1: Subject characteristics during breathing a gas mixture with $19.8 \%$ and $14.8 \% \mathrm{O}_{2}$. Values shown are mean \pm standard deviation. $p$-values were estimated between two stages of the experimental procedure. ${ }^{*} \mathrm{p}<0.05 ;{ }^{* *} \mathrm{p}<0.01 ;{ }^{* * *} \mathrm{p}<0.001$. Used abbreviation: HR - heart rate, DBP - diastolic blood pressure, SBP - systolic blood pressure, MAP - mean arterial pressure, SAS LEFT - subarachnoid width, $\mathrm{HbO}_{2}$ RIGHT - relative changes in oxyhaemoglobin, $\mathrm{tHb}$ - relative changes in total haemoglobin $\mathrm{SaO}_{2}$ - oxyhaemoglobin saturation, $\mathrm{EtCO}_{2}$ - end-tidal $\mathrm{CO}_{2}, \mathrm{EtO}_{2}$ - end-tidal $\mathrm{O}_{2}$. 
Figure 1 shows the result of the amplitude of the wavelet transform (WT) for one representative volunteer. Left and right columns of Fig. 1 corresponds to breathing a gas mixture with $19.8 \%$ and $14.8 \% \mathrm{O}_{2}$, respectively. We estimated WT for all four measured signals: BP (a, e), ECG (b, f), $\mathrm{HbO}_{2 \text { RIGHT }}(\mathbf{c}, \mathbf{g})$ and SASLEFT $(\mathbf{d}, \mathbf{h})$. Cardiac oscillations $(\sim 1 \mathrm{~Hz})$ are clearly visible for the whole duration of all signals. Additionally, collected signals (excluding ECG) manifest over a wide frequency range, but SASLEFT and $\mathrm{HbO}_{2}$ RIGHT signals have more prominent low frequency components than BP signal (Gruszecka et al. 2020).

To simplify the comparison between two stages of experimental procedure in terms of their frequency content, we plotted the median of the time-averaged amplitude of wavelet transforms (Figure 2). In this plot, we added six different frequency intervals which correspond to different physiological functions described previously by Stefanovska et al. 1999 and Gruszecki et al. 2018. Additionally, we estimated the pvalue of the differences between the results for breathing a gas mixture with $19.8 \%$ and $14.8 \% \mathrm{O}_{2}$ for all measured signals. We observed the highest differences $(p<0.001)$ for WT amplitude for SASLEFT for all frequency intervals. For $\mathrm{HbO}_{2}$ RIGHT the differences between amplitude of wavelet transform were associated with respiration (II, 0.145$0.6 \mathrm{~Hz}$ ), neurogenic (IV, $0.021-0.052 \mathrm{~Hz}$ ), endothelial nitric oxide (NO) dependent (V, 0.0095-0.021) and NO independent ( $\mathrm{VI}, 0.005-0.0095 \mathrm{~Hz}$ ). In turn, WT amplitude of the BP signal only has differences $(p<0.05)$ in myogenic $(I I I, 0.052-0.145 \mathrm{~Hz})$ and neurogenic (IV) intervals. We did not observe any statistically significant differences for the ECG signal. 

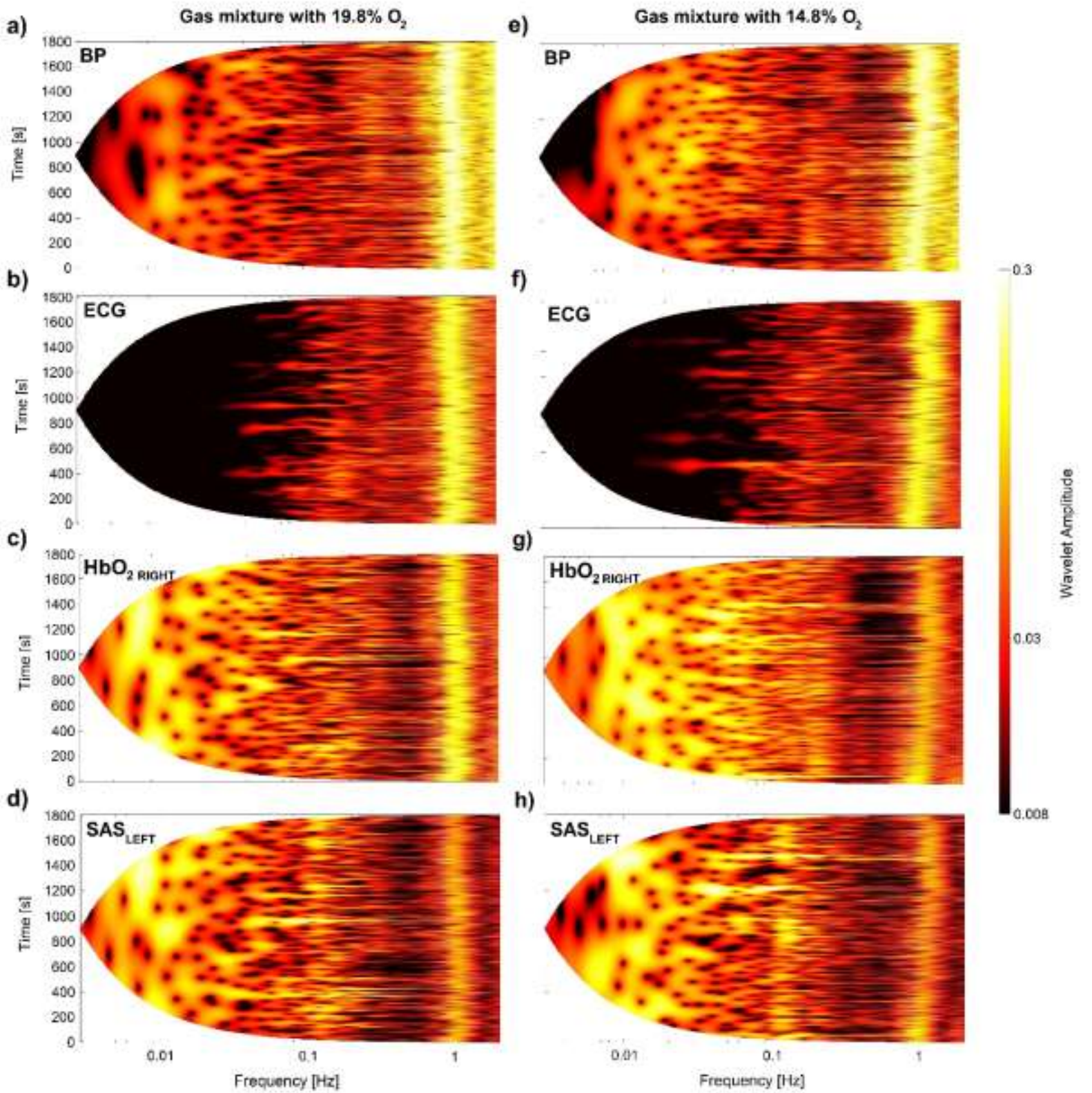

Figure 1: Wavelet transform of recorded signals: $B P$ ( $\boldsymbol{a}$ and $\boldsymbol{e}$ ), ECG (b and $\boldsymbol{f}$ ), $\mathrm{HbO}_{2 \mathrm{RIGHT}}$ (c and $\boldsymbol{g}$ ) and $S A S_{\text {LEFT }}(\boldsymbol{d}$ and $\boldsymbol{h}$ ) for one of the volunteers. Left (right) columns of plots illustrate results for breathing a gas mixture with $19.8 \%(14.8 \%) \mathrm{O}_{2}$.

Figure 3 illustrates the wavelet phase coherence (left column) and phase difference (right column) between pairs of all collected signals. The value of phase coherence was significant when the value of phase coherence was higher than $95^{\text {th }}$ percentile of 435 (2-permutation of 30 subjects) inter-subject surrogate. When we observed statistically significant phase coherence at certain frequencies, we assumed the same for phase differences. For phase coherence we found statistically significant differences for BP-SASLEFT and BP-ECG only for the cardiac interval. We did not observe any statistically significant difference for phase difference for all considered pairs of signals. 


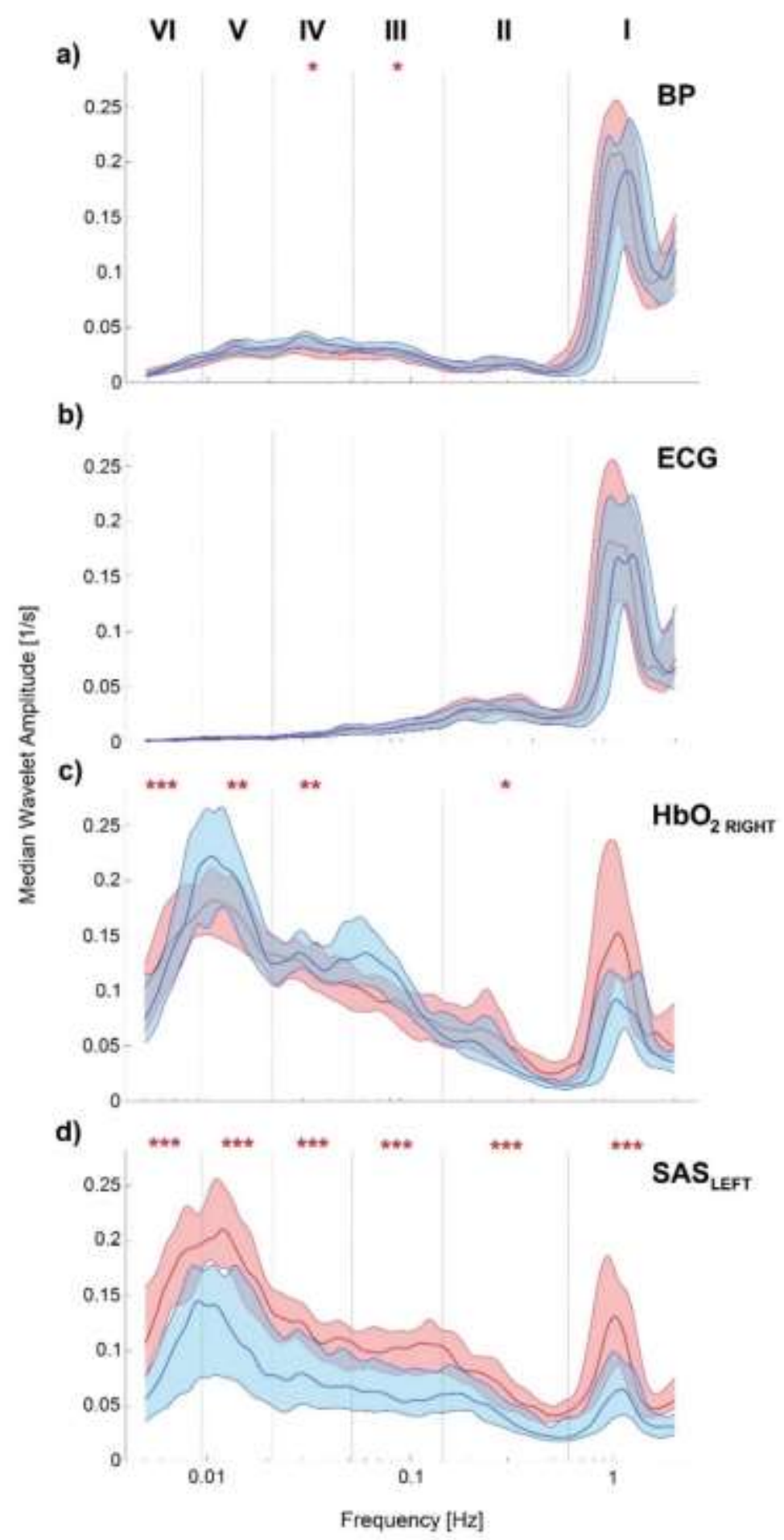

Figure 2: Median (thick lines) of the time-averaged wavelet transforms of signals recorded in all 30 subjects: (a) $B P$, (b) $E C G$, (c) $\mathrm{HbO}_{2}$ RIGHT and (d) SAS LEFT obtained from the 30 minutes continuous recordings. Shaded areas indicate the inter-quartile range (25th, 75th percentiles). Red (blue) lines and shade areas correspond to breathing a gas mixture with $19.8 \%(14.8 \%) O_{2} \cdot{ }^{*} p<0.05 ;{ }^{* *} p<0.01 ;{ }^{* * *} p$ $<0.001$. 

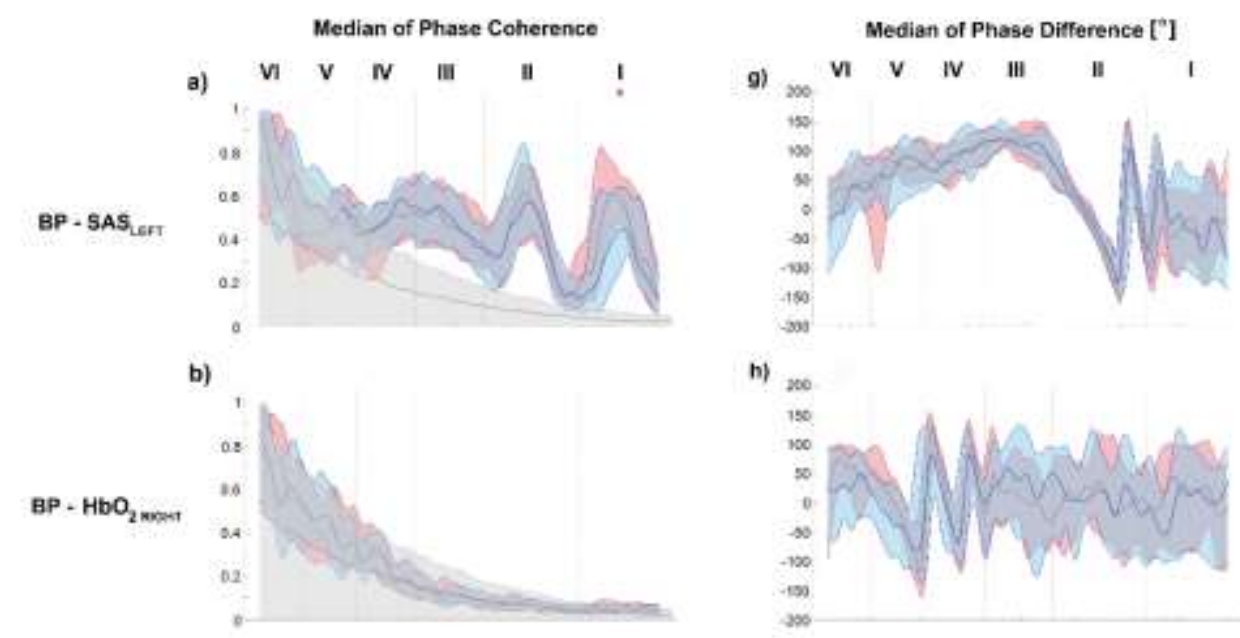

h)
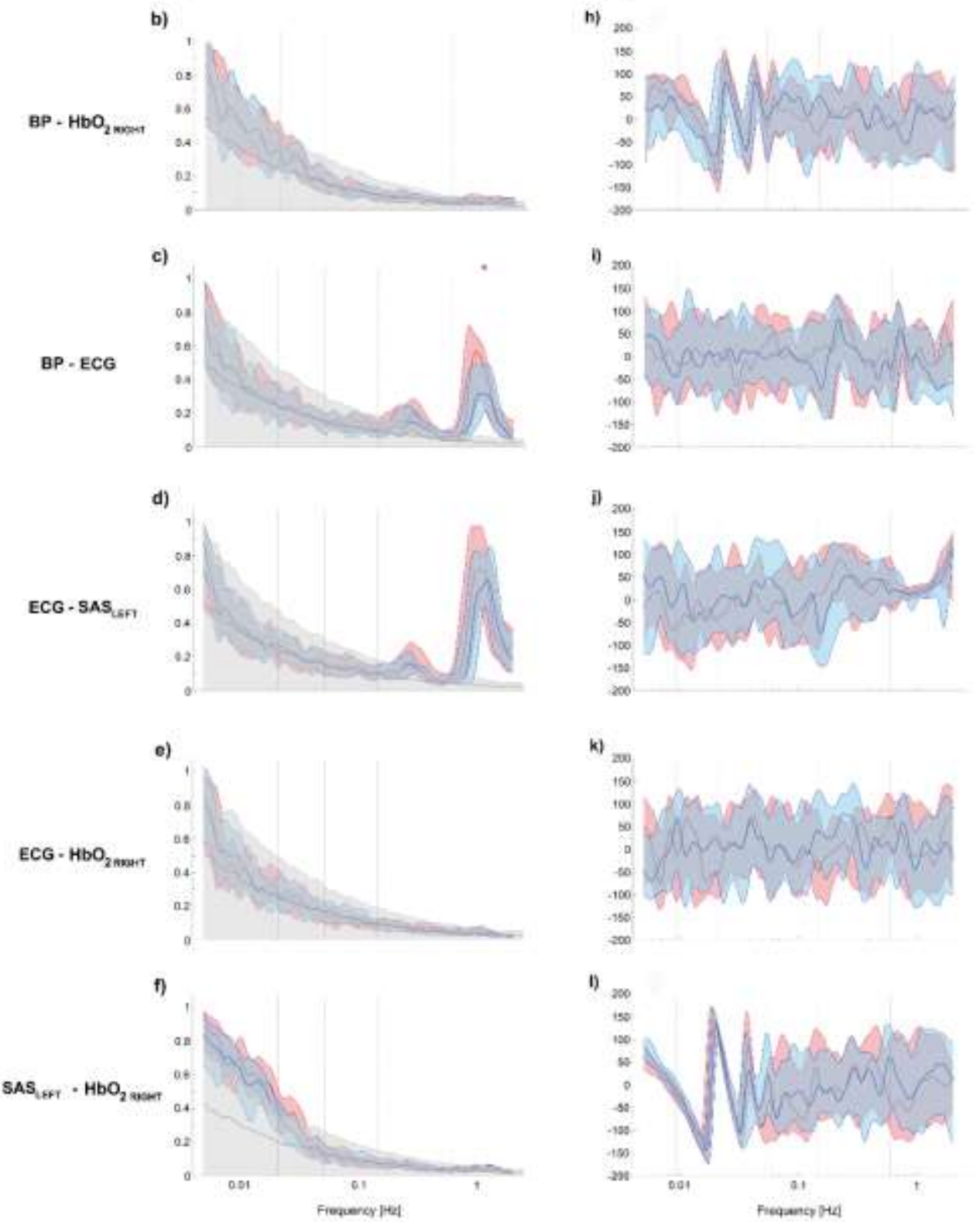

Figure 3: Median (thick lines) of wavelet phase coherence (left column) and phase difference (right column) between $(\boldsymbol{a}, \boldsymbol{g}) B P$ vs SAS LEFT, $(\boldsymbol{b}, \boldsymbol{h}) B P$ vs $\mathrm{HbO}_{2}$, (c, i) BP vs ECG, $(\boldsymbol{d}, \boldsymbol{j})$ ECG vs SAS LEFT, $_{\text {, }}(\boldsymbol{e}, \boldsymbol{k})$ ECG vs $\mathrm{HbO}_{2}$ and $(f, \mathrm{l}) \mathrm{SAS}$ LEFT vs $\mathrm{HbO}_{2}$. Coloured shading indicates the interquartile range (25th, 75th percentiles) for 30 subjects. Coherence below the 95th percentile of the surrogates (light grey line and shading) is not considered significant. Red (blue) lines and shade areas correspond to breathing a gas mixture with $19.8 \%(14.8 \%) 0_{2 .}{ }^{*} p<0.05 ;{ }^{* *} p<0.01 ;{ }^{* *} p<0.001$. 


\section{Discussion}

The novel findings of our study showed that mild poikilocapnic hypoxia increases the very low frequency $\mathrm{HbO}_{2}$ oscillations $(<0.052 \mathrm{~Hz})$ in prefrontal cortex while diminishing SAS oscillations in the whole frequency range. In addition we confirmed that prefrontal cortex $\mathrm{HbO}_{2}$ and SAS width decline during exposure to lowered oxygen partial pressure.

In recent years there has been an increased interest in cerebrovascular mechanisms related to normal and pathological physiology. As such, we have witnessed a substantial paradigm shift in our thinking in regard to brain perfusion control mechanisms. The increasing accumulation of evidence suggests that astrocytes act as physiological sensors reacting to changes in the brain parenchymal levels of metabolic substrates (such as oxygen), cerebral perfusion and intracranial pressure changes (Angelova et al. 2015, Marina et al. 2018, Turovsky et al. 2020, Marina et al. 2020). In particular, astrocytes might be responsible for local cellular signalling mechanisms adjusting cerebral perfusion to brain metabolic needs (Marina et al. 2020).

Our study strongly supports the view that local brain mechanisms, rather than systemic control-level mechanisms are responsible for adaptation to mild poikilocapnic (normoxic) hypoxia. We observed decreases in respiratory contribution to $\mathrm{HbO}_{2}$ prefrontal cortex oscillations, while at the same time observing substantially increased very low frequency $\mathrm{HbO}_{2}$ oscillations $(<0.052 \mathrm{~Hz})$. Very low frequency periodic dynamism is believed to reflect neural sympathetic and metabolic activity (Saleem et al. 2016, Chow et al. 2007), and is most likely linked to increased neural metabolism (Vestergaard et al. 2016).

Prefrontal cortex $\mathrm{HbO}_{2}$ fluctuations at the $0.145-0.052 \mathrm{~Hz}$ frequency were reported to be linked to general BP oscillations (van Beek et al. 2012, Vermeij et al. 2014, Yunjie et al. 2010). In response to normobaric hypoxia, BP fluctuations did not change in the $0.145-0.052$ frequency. We also did not observe any phase difference in the $\mathrm{HbO}_{2}$ and BP signals. Stabilisation of BP-brain signals oscillations at the $0.145-0.052 \mathrm{~Hz}$ frequency are most likely driven by increased sympathetic activity related to hypoxia and slightly increased intracranial pressure (Talbot et al. 2005, Dubowitz et al. 2009, 
DiPasquale et al. 2016). We have previously shown that high sympathetic drive strengthen the links between BP and SAS signal (Winklewski et al. 2019).

Slightly increased intracranial pressure and discrete brain swelling previously reported in hypoxia could have resulted in a decline of SAS oscillations across the whole frequency range. Although we cannot confirm discrete brain swelling from our study, our findings extend previous results collected in our laboratory where healthy subjects were exposed to 5 minutes of poikilokapnic (normobaric) hypoxia, and we observed a trend towards a decline in SAS width and diminished BP-SAS amplitude coupling (Wszedybyl-Winklewska et al. 2017). In the current study, we observed diminished BPSAS and ECG-BP phase coherences within the cardiac frequency band. Suppression of respiratory SAS oscillations were reported previously by our team as an early indicator of rising intracranial pressure in rabbit (Frydrychowski et al. 2011). A decline in the modulation of cerebral blood flow by even moderately increased intracranial pressure was also observed in humans (Haubrich et al. 2007).

This study used poikilocapnic (normobaric) hypoxia to challenge whether $\mathrm{EtCO}_{2}$ fluctuations varied in response to reflexive hyperventilation. We preferred this over an isocapnic hypoxic challenge, defined by hypoxia in the presence of maintained $\mathrm{CO}_{2}$ within the bloodstream, as we believe our chosen methodology better mimicked clinical conditions. A gas mixture of $19.8 \% \mathrm{O}_{2}$ was used as the normoxic condition as this is the native partial pressure of oxygen in Regina, Saskatchewan, Canada (577 meters above sea level) where the testing was performed.

Our study demonstrates that clinical situations associated with even mild cerebral hypoxia may result in several pathophysiological adaptations including changes in brain metabolism and cerebrospinal fluid dynamics. Non-invasive modalities that enable continuous brain monitoring could provide several benefits to patients suffering from diseases associated with declined brain oxygenation, stroke or traumatic brain injury.

\section{Conclusions}

In subjects exposed to mild poikilokapnic hypoxia we have demonstrated for the first time that: 1) very low $\mathrm{HbO}_{2}$ oscillations $(<0.052 \mathrm{~Hz}$ ) increase in prefrontal cortex, and 2) SAS fluctuation diminishes in the whole frequency range. Consequently the study 
provides insights into the physiological mechanisms governing brain response to a mild hypoxic challenge. Our study supports the notion that $\mathrm{HbO}_{2}$ and $\mathrm{SAS}$ width monitoring might be beneficial for patients with acute lung disease, including SARS-CoV-2.

\section{Materials and Methods}

Subjects

A group of 30 healthy and not smoking volunteers (28 males and 2 females, age $30.8 \pm 13.4$ years, $\mathrm{BMI}=24.5 \pm 2.3 \mathrm{~kg} / \mathrm{m}^{2}$ ) were involved in the experiments, which were carried out in accordance with the recommendations of Helsinki. The Ethics Committee of University of Regina (REB\#2017-013) approved this study and the experimental protocol. All subjects were older than 18 years and signed a consent form to participate in the study. Participants were asked to avoid any alcohol at least 24 hours before the experiment, and tea, coffee, nicotine, cocoa and any food and beverages containing methylxanthine for at least 12 hours before the experiment. Intense exercise training was not allowed at least 6 hours prior to testing, and all subjects were asked to void their bladder within 30 minutes of testing. All procedures were preceded by 10 minutes of rest in the sitting position in a comfortable chair located in quiet room.

\section{Experimental design}

Two tests of breathing a gas mixture with $19.8 \%$ and $14.8 \% \mathrm{O}_{2}$ were conducted in a quiet room and environmental chamber. Room and chamber were pre-set to a temperature of $18-20^{\circ} \mathrm{C}$. After all of the medical research equipment was attached to the volunteer participant (see Measurements below), they were instructed to lie supine on a bed with a pillow to support their head during breathing a gas mixture of normal room air $\left(19.8 \% \mathrm{O}_{2}\right)$ and during normobaric hypoxia $\left(14.8 \% \mathrm{O}_{2}\right)$. The first 30 minutes of experiment, subjects were asked to breath room air $\left(19.8 \% \mathrm{O}_{2}\right)$, and then they moved to the environmental chamber with the aid of a research assistant (it took about 3-5 minutes). During the next 30 minutes, volunteer subjects breathed freely in the hypoxic chamber with reduced levels of oxygen (14.8\%). The hypoxic oxygen condition mimiced the elevation of approximately 2900-3000 meters above sea level.

\section{Measurements}

A Finometer (Finapres Medical Systems, Arnhem, The Netherlands) was used to measure heart rate $(\mathrm{HR})$ and BP. Finger photoplethysmography BP was calibrated 
against brachial arterial pressure. The signal was collected from the left middle finger at all times during both normoxia $\left(19.8 \% \mathrm{O}_{2}\right)$ and hypoxia $\left(14.8 \% \mathrm{O}_{2}\right)$. Oxyhaemoglobin saturation $\left(\mathrm{SaO}_{2}\right)$ was measured using a Nellcor PM10N Portable $\mathrm{SpO}_{2}$ Patient Monitoring System (Medtronic Canada, Vancouver, BC). During the experiment, the device was placed on the right index finger. Expired respiratory gas samples from the mouthpiece were constantly analysed using the side-stream technique for end-tidal $\mathrm{CO}_{2}\left(\mathrm{EtCO}_{2}\right)$ using AD Instruments ML206 gas analyser (Colorado Springs, USA). SAS signal was collected using an SAS Monitor (NIRTI SA, Wierzbice, Poland). A detailed description of the SAS Monitor has been provided previously (e.g. Gruszecki et al. 2018). The NIRS signal was collected by PortaLite system (Artinis Medical, The Netherlands). The sensor was positioned over pre-frontal cortex of the right hemisphere during the experiment. NIRS contains transmitters at 30,35 and $40 \mathrm{~mm}$ from the receiver, which allows a penetration depth of approximately one-third to onehalf of the distance between optodes (Cui et al. 1991). During the experiment, we collected the following NIRS signals: relative changes in oxy- $\left(\mathrm{HbO}_{2}\right)$, deoxy- $(\mathrm{HHb})$, total haemoglobin $\left(\mathrm{tHb}=\mathrm{HbO}_{2}+\mathrm{HHb}\right)$ and haemoglobin difference $\left(\mathrm{Hb}_{\text {diff }}=\mathrm{HbO}_{2}\right.$ $\mathrm{HHb}$ ). To avoid admission of background light the NIRS sensor was secured with a black coloured tensor bandage. We decided to analyse a NIRS signal that penetrate the deepest regions of head to be sure that we truly analyse signals from brain vessels. Like many others, (Bernjak et al. 2012, Cui et al. 2014, Kim et al. 2020) we focused our analyses on the oxyhaemoglobin $\left(\mathrm{HbO}_{2}\right)$ signal. Additionally, to avoid any interference between NIRS and SAS signals, we recorded only SASLEFT from left hemisphere.

All parameters were recorded and saved simultaneously for further analysis. This was completed for both segments, with the first 30 minute normoxic condition completed first, followed by the subsequent 30 minute normobaric hypoxic second. To import and view collected signals, a PowerLab 8/32 amplifier was coupled with LabChart 7 Pro (AD Instruments, Colorado Springs, Colorado, USA). All signals before analysis were down sampled to $10 \mathrm{~Hz}$, detrended using a moving average with a window size of 220 seconds, and normalized by subtraction of their mean and division by their standard deviation. 


\section{Wavelet transform}

To detect and perform analysis of physiological processes that are responsible for generating oscillations in the cardiovascular system, we used wavelet analysis. The wavelet transform is a method that transforms a signal from the time domain to the time-frequency domain. The definition of the wavelet transform is:

$$
W(s, t)=\frac{1}{\sqrt{s}} \int_{-\infty}^{+\infty} \varphi\left(\frac{u-t}{s}\right) g(u) d u,
$$

where $W(s, t)$ is the wavelet coefficient, $g(u)$ is the time series and $\varphi$ is the Morlet mother wavelet, scaled by factor $s$ and translated in time by $t$. The Morlet mother wavelet is defined by the equation:

$$
\varphi(u)=\frac{1}{\sqrt[4]{\pi}} \exp (-i 2 \pi u) \exp \left(-0.5 u^{2}\right),
$$

where $i=\sqrt{-1}$. The rationale for using the Morlet wavelet is that it affords good localization of events in time and frequency due to its Gaussian shape (Bernjak et al. 2012). The wavelet coefficients are complex numbers in the time-frequency plane when the Morlet wavelet is used:

$$
X\left(\omega_{k}, t_{n}\right)=X_{k, n}=a_{k, n}+i b_{k, n} .
$$

They define the instantaneous relative phase,

$$
\theta_{k, n}=\arctan \left(\frac{b_{k, n}}{a_{k, n}}\right),
$$

and the absolute amplitude,

$$
\left|X_{k, n}\right|=\sqrt{a_{k, n}^{2}+b_{k, n}^{2}},
$$

for each frequency and time.

During the measurement, hypoxia may create phase modulations. A mathematical tool to find the relationship between the phases of two signals is the wavelet phase coherence (WPCO). WPCO enables us to determine whether the oscillations detected are significantly correlated over time. To estimate the WPCO we used the following expression (Lachaux et al. 2002):

$$
C_{\theta}\left(f_{k}\right)=\frac{1}{n}\left|\sum_{t=1}^{n} \exp \left[i\left(\theta_{2 k, n}-\theta_{1 k, n}\right)\right]\right|,
$$


where $\theta_{k, n}=\arctan \left(\frac{b_{k, n}}{a_{k, n}}\right)$ is an instantaneous measure of phases at each time $\mathrm{t}_{\mathrm{n}}$ and frequency $f_{k}$ for both signals. When two oscillations are unrelated (related), their phase difference continuously changes (remain constant) with time, thus their $C_{\theta}\left(f_{k}\right)$ approaches zero (one).

Additionally, we can calculate the phase difference $\Delta \theta_{k}$, which provides information about the phase lag of one oscillator compared to the other:

$$
\Delta \theta_{k}=\arctan \left(\frac{\frac{1}{n} \sum_{t=1}^{n} \sin \left(\Delta \theta_{2 k, n}-\Delta \theta_{1 k, n}\right)}{\frac{1}{n} \sum_{t=1}^{n} \cos \left(\Delta \theta_{2 k, n}-\Delta \theta_{1 k, n}\right)}\right),
$$

where $\Delta \theta_{k} \in\left(-180^{\circ}, 180^{\circ}\right)$.

\section{Statistical analysis}

To avoid the assumption of normality in the results, nonparametric statistical tests were used for all comparisons. The Wilcoxon rank sum test was used to compare whether the median of results for breathing a gas mixture with $19.8 \% \mathrm{O}_{2}$ and $14.8 \%$ $\mathrm{O}_{2}$ was significantly different. The results of our calculations are found in Tables 1 and Figures 2, and 3.

To test whether the estimated values of phase coherence are statistically significant, the surrogate data testing method was used (Lancaster et al. 2018). As we know there are naturally less cycles of oscillations the lower in frequency that we consider. This can cause artificially increased wavelet phase coherence at low frequencies, even in cases where there is none. The surrogate analysis helps us to find a significance level above which the phase coherence may be regarded as physically meaningful. To estimate significance level, we used intersubject surrogates (Sun et al. 2012), which assumes that the signals collected from different subjects must be independent, while having similar characteristic properties. The actual value of phase coherence obtained at each frequency can then be compared with the surrogate threshold. When the phase coherence is located above the threshold it is considered to be statistically significant.

\section{Funding}

Dr Marcin Gruszecki was supported by the NCN-grant number 2018/02/X/NZ4/00464. 


\section{Acknowledgements}

We would like to thank all of the volunteer participants that contributed their valuable time to these experiments.

\section{Competing interests}

Profs Andrzej F. Frydrychowski and Pawel J. Winklewski are stakeholders in NIRTI SA. The other authors declare no competing interests.

\section{Authors' contributions}

Conceived and designed the experiments: A.G., M.G., J.P.N., P.J.W. Performed the experiments: J.S., T.T., M.G. Analysed the data: A.G. M.G. Contributed reagents/materials/analysis tools: M.A.M., P.N., G.P.K., M.W.W., A.F., J.R., N.G., A.G., P.L., M.G. Co-wrote the paper: A.G., M.G., P.J.W. All authors reviewed the manuscript and agreed to publish the paper.

\section{Data Availability Statement}

The data presented in this study are available on request from the corresponding author. The data are not publicly available due to privacy.

\section{References}

Alonso-Lana, S., Marquié, M., Ruiz, A., Boada, M. Cognitive and Neuropsychiatric Manifestations of COVID-19 and Effects on Elderly Individuals With Dementia, Front. Aging Neurosci. 2020; 12, 588872

Hampson, N.B., Camporesi, E.M., Stolp, B.W., Moon, R.E., Shook, J.E., Griebel, J.A., and Piantadosi, C.A., Cerebral oxygen availability by NIR spectroscopy during transient hypoxia in humans J Appl Physiol. 1990; 69(3):907-13

Kety, S.S., Schmidt, C.F. The effects of altered arterial tensions of carbon dioxide and oxygen on cerebral blood flow and cerebral oxygen consumption of normal young men J. Clin. Invest. 1948; 27(4): 484-92

Dubowitz, D.J., Dyer, E.A.W., Theilmann, R.J., Buxton, R.B. and Hopkins, S.R. Early brain swelling in acute hypoxia, J Appl Physiol 2009; 107:244-252 
DiPasquale, D.M., Muza, S.R., Gunn, A.M., Li, Z., Zhang, Q., Harris, N.S., Strangman, G.E. Evidence for cerebral edema, cerebral perfusion, andintracranial pressure elevations in acute mountain sickness 2016, Brain and Behavior; 6(3), e00437 Halder, S.K., Milner, R. Mild hypoxia triggers transient blood-brain barrier disruption: a fundamental protective role for microglia, Acta Neuropath. Communications 2020; 8:175 Vestergaard, M.B., Lindberg, U., Aachmann-Andersen, N.J., Lisbjerg, K., Christensen, S.J., Law, I., Rasmussen, P., Olsen, N.V., Larsson, H.B.V. Acute hypoxia increases the cerebral metabolic rate - a magnetic resonance imaging study 2016; 36(6):1046-58

Saleem, S., Teal, P.D., Kleijn W.B., Ainslie, P.N., Tzeng, Y.C. Identification of human sympathetic neurovascular control using multivariate wavelet decomposition analysis Am J Physiol Heart Circ Physiol. 2016 ;311(3):H837-48

Marina, N., Christie, I.N., Korsak, A., Doronin, M., Brazhe, A., Hosford, P.S., Wells, J.A., Sheikhbahaei, S., Humoud, I., Paton, J.F.R., Lythgoe, M.F., Semyanov, A., Kasparov S.,. Gourine, A.V. Astrocytes monitor cerebral perfusion and control systemic circulation to maintain brain blood flow 2020, Nature Communications, $11: 131$

Winklewski, P.J., Wolf, J., Gruszecki, M., Wszedybyl-Winklewska, M., Narkiewicz, K. Current understanding of the effects of inspiratory resistance on the interactions between systemic blood pressure, cerebral perfusion, intracranial pressure, and cerebrospinal fluid dynamics. J Appl Physiol 2019, 127: 1206-1214

Ferrari, M., Quaresima, V. A brief review on the history of human functional nearinfrared spectroscopy (fNIRS) development and fields of application Neuroimage, 2012; 63(2):921-35

Gruszecki, M., Nuckowska, M.K., Szarmach, A., Radkowski, M., Szalewska, D., Waskow, M., Szurowska, E., Frydrychowski, A.F., Demkow, U., Winklewski, P.J. 
Oscillations of Subarachnoid Space Width as a Potential Marker of Cerebrospinal Fluid Pulsatility Adv. Exp. Med. Biol. 2018; 1070:37-47

Frydrychowski, A., Gumiński, W., Rojewski, M., Kaczmarek, J.W., Juzwa, W. Technical foundations for noninvasive assessment of changes in the width of the subarachnoid space with near-infrared transillumination-backscattering sounding (NIR-TBSS). leee Transactions On Biomedical Engineering 2002; 49(8):887-904 Stefanovska, A., Bracic, M., Kvernmo, H.D. Wavelet analysis of oscillations in the peripheral blood circulation measured by laser Doppler technique IEEE Transactions on Biomedical Engineering 1999; 46(10):1230-9

Finch CA, Lenfant C. Oxygen transport in man. N Engl J Med 1972; 286(8):407-415 Talbot NP, Balanos GM, Dorrington KL, Robbins PA. Two temporal components within the human pulmonary vascular response to approximately $2 \mathrm{~h}$ of isocapnic hypoxia. J Appl Physiol. 2005; 98, 1125

Dubowitz DJ, Dyer EA, Theilmann RJ, Buxton RB, Hopkins SR. Early brain swelling in acute hypoxia. J. Appl Physiol. 2009; 107: 244-52

Greitz D, Franck A, Nordell B. On the pulsatile nature of intracranial and spinal CSF-circulation demonstrated by MR imaging. Acta Radiol. 1993; 34: 321

Linninger AA, Tsakiris C, Zhu DC, Xenos M, Roycewicz P, Danziger Z, et al. Pulsatile cerebrospinal fluid dynamics in the human brain. IEEE Trans Biomed Eng. 2005; 52: $557-565$.

Gruszecki, M. et al. Human subarachnoid space width oscillations in the resting state. Sci. Rep. 8, 3057 (2018)

Gruszecka A. et al. Comparison of near infrared spectroscopy (NIRS) and nearinfrared transillumination-backscattering sounding (NIR-T/BSS) methods. Sci. Rep. 10(1), 18668 (2020)

Bernjak, A. et al. Coherence between fluctuations in blood flow and oxygen saturation. Fluct. Noise Lett. 11, 1-12 (2012).

Lachaux, J. P. et al. Estimating the time-course of coherence between single-trial brain signals: an introduction to wavelet coherence. 
Clin. Neurophysiol. 32, 157 (2002).

Lancaster, G. et al. Surrogate data for hypothesis testing of physical systems. Physics Rep. 748, 1-60, (2018).

Sun, J., Hong, X. \& Tong, S. Phase synchronization analysis of EEG signals: an evaluation based on surrogate tests. IEEE Trans. Biomed. Eng. 59, 2254-2263 (2012).

Stefanovska A, Bračič M, Kvernmo HD. Wavelet analysis of oscillations in the peripheral blood circulation measured by laser Doppler technique, IEEE Trans. Bio. Med. Eng., 1999;46:1230-1239.

Cui, W., Kumar, C. \& Chance, B. Experimental study of migration depths for the photons measured at sample surface. SPIE 1431, 180-191 (1991)

Bernjak, A., Stefanovska, A. \& McClintock, P. V. E. Coherence between fluctuations in blood flow and oxygen saturation. Fluct. Noise Lett. 11, 1-12 (2012)

Cui, R. et al. Wavelet coherence analysis of spontaneous oscillations in cerebral tissue oxyhaemoglobin concentrations and arterial blood pressure in elderly subjects. Microvasc Res. 93, 14-20 (2014).

Kim, T. J. et al. Prognostication of neurological outcome after cardiac arrest using wavelet phase coherence analysis of cerebral oxygen. Resuscitation 150, 41-49 (2020)

Wszedybyl-Winklewska, M., Wolf, J., Świerblewska, E., Kunicka, K., Gruszecka, A., Gruszecki, M., Kucharska, W., Winklewski, P.J., Zabulewicz, J., Gumiński, W., Pietrewicz, M., Frydrychowski, A.F., Bieniaszewski, L., Narkiewicz, K. Acute hypoxia diminishes the relationship between blood pressure and subarachnoid space width oscillations at the human cardiac frequency. 2017, Plos One, 12(2), 1-13

Baumann, R., Bauer, Ch., and Bartels, H., Sixth Intern. Berlin Symp. Structure and Function of Erythrocytes, August 19-22 (1970)

Lenfant, C. T., Torrance, E., Englisch, E., Finch, C. A., Reynafafje, C., Ramos, J., and Faura, J., J. Clin. Invest., 47, 2652 (1968)

Dubowitz DJ, Dyer EA, Theilmann RJ, Buxton RB, Hopkins SR. Early brain swelling in acute hypoxia. J. Appl Physiol. 2009; 107: 244

DiPasquale DM, Muza SR, Gunn AM, Li Z, Zhang Q, Harris NS, et al. Evidence for cerebral edema, cerebral perfusion, and intracranial pressure elevations in acute mountain sickness. Brain Behav. 2016; 6: e00437 
Michiels, C., Arnould, T., Remacle, J., Endothelial cell responses to hypoxia: initiation of a cascade of cellular interactions, Biochimica et Biophysica Acta, 2000, 1497(1), 110

Angelova, P.R., Kasymov, V., Christie, I., Sheikhbahaei, S., Turovsky, E., Marina, N., Korsak, A., Zwicker, J., Teschemacher, A.G., Ackland, G.L., Funk, G.D., Kasparov, S., Abramov A.Y., Gourine, A.V. Functional Oxygen Sensitivity of Astrocytes Journal of Neuroscience 2015; 35(29):10460-10473

Marina, N., Turovsky, E., Christie, I.N., Hosford, P.S., Hadjihambi, A., Korsak, A., Ang, R., Mastitskaya, S., Sheikhbahaei, S., Theparambil, S.M., Gourine, A.V. Brain metabolic sensing and metabolic signaling at the level of an astrocyte Glia 2018; 66(6):1185-1199

Turovsky, E.A., Braga, A., Yu, Y., Esteras, N., Korsak, A., Theparambil, S.M., Hadjihambi, A., Hosford, P.S., Teschemacher, A.G., Marina, N., Lythgoe, M.F., Haydon P.G., Gourine, A.V. Mechanosensory Signaling in Astrocytes Journal of Neuroscience 2020; 40(49):9364-9371

Chow, N., Bell, R.D., Deane, R., Streb, J.W., Chen, J., Brooks, A., Nostrand, W.V., Miano, J.M., Zlokovic, B.V. Serum response factor and myocardin mediate arterial hypercontractility and cerebral blood flow dysregulation in Alzheimer's phenotype PNAS 2007; 104(3):823-828

van Beek, A.H.E.A., Lagro, J., Olde-Rikkert, M.G.M., Zhang, R., Claassen, J.A.H.R. Oscillations in cerebral blood flow and cortical oxygenation in Alzheimer's disease Neurobiol Aging 2012; 33(2):428.e21-31

Vermeij, A., Meel-van den Abeelen, A.S.S., Kessels, R.P.C., van Beek, A.H.E.A., Claassen, J.A.H.R. Very-low-frequency oscillations of cerebral hemodynamics and blood pressure are affected by aging and cognitive load Neurolmage 2014; 85:608615

Yunjie, T., Blaise Deb, F. Time lag dependent multimodal processing of concurrent fMRI and near-infrared spectroscopy (NIRS) data suggests a global circulatory origin for low-frequency oscillation signals in human brain Neuroimage 2010; 53(2):553-64 Frydrychowski, A.F., Wszedybyl-Winklewska, M., Guminski, W., Przyborska, A., Kaczmarek, J., Winklewski, P.J. Use of Near Infrared Transillumination / Back Scattering Sounding (NIR-T/BSS) to assess effects of elevated intracranial pressure on width of subarachnoid space and cerebrovascular pulsation in animals Acta Neurobiol Exp. 2011; 71(3):313-21 
Haubrich, C., Czosnyka, Z., Lavinio, A., Smielewski, P., Diehl, R.R., Pickard, J.D., Czosnyka, M. Is there a direct link between cerebrovascular activity and cerebrospinal fluid pressure-volume compensation? Stroke, 2007; 38(10):2677-80 